\title{
Numerical Solutions of Steady Tensorial Transport Equations Using Discontinuous Galerkin Method Implemented in FreeFem++
}

\author{
K. M. Helal" \\ Department of Mathematics, Comilla University, Comilla, Bangladesh \\ Received 10 September 2015, accepted in final revised form 11 October 2015
}

\begin{abstract}
The main purpose of this paper is to approximate the solution of the steady tensorial transport equations using discontinuous Galerkin finite element method implemented with the finite element solver FreeFem++. After introducing the formulations of the tensorial transport equations, the analysis of its componentwise equations, i.e., advection-reaction equations have been discussed. Discretizing the transport problem using discontinuous Galerkin finite element method, the iterative fixed-point method is used to obtain the solutions. We present the numerical simulations of two-dimensional benchmark problem and observe the instability of elasticity. All the simulations are done using the script developed in FreeFem++.
\end{abstract}

Keywords: Transport equations; Finite element method; Discontinuous Galerkin method; FreeFem++.

(C) 2016 JSR Publications. ISSN: 2070-0237 (Print); 2070-0245 (Online). All rights reserved. doi: http://dx.doi.org/10.3329/jsr.v8i1.24960

J. Sci. Res. 8 (1), 29-39 (2016)

\section{Introduction}

This paper aims to approximate the solution of the steady tensorial transport equations using discontinuous Galerkin (DG) finite element method. The unknown is the viscoelastic extra stress tensor $\sigma$. An iterative method based on the application of a fixed point algorithm is implemented to solve the steady tensorial transport equations which are discretized using the discontinuous Galerkin finite element. The finite element solver FreeFem++ is used to obtain the solution with the implementation of the above method. The discontinuous $\mathbb{P}_{1}$ elements $\left(\mathbb{P}_{1} d c\right)$ are used to discretize the transport problem, and to obtain the numerical solutions of the corresponding algebraic system of the transport equations. The numerical results are obtained both

\footnotetext{
Corresponding author: khalifahelal@gmail.com
} 
computationally and graphically by the implementation of DG finite element method in FreeFem++ [1].

I first introduce the steady tensorial transport equation which is the non-linear system of partial differential equations of hyperbolic type. We split it into componentwise scalar equation which can be identified as the hyperbolic equations known as advection-reaction equations. We briefly discuss the mathematical and numerical analysis, and analyze the approach problem in the context of discontinuous Galerkin finite element method [2-4]. An auxiliary problem which is in the form of transport problem with known vector field for velocity is considered. Using the variational formulation of discrete transport equations, the dimensionless transport problem is written in terms of components as a system of three scalar transport equations (adevection-reaction equations). Then an iterative method is used to solve the system. The linear system is solved using the default solver sparse-solver [1]. All the numerical simulations are implemented with our own script developed in FreeFem++. Finally, we draw some conclusions.

\section{Nomenclature}

Before discussing the mathematical analysis of tensorial transport equations with boundary conditions and variational formulation, we introduce some notations of different function spaces in the following table, details of which can be found in $[5,6]$.

\begin{tabular}{|c|c|}
\hline$L^{p}(\Omega)$ & The Lebesgue spaces \\
\hline $\begin{array}{l}\mathrm{W}^{\mathrm{m}, \mathrm{p}}(\Omega) \text { where } \mathrm{m} \geq 0 \text { be an } \\
\text { integer and } 1 \leq \mathrm{p} \leq \infty\end{array}$ & The standard Sobolev spaces \\
\hline$\|\cdot\|_{m, p}$ & Norms of $\mathrm{W}^{\mathrm{m}, \mathrm{p}}(\Omega)$ \\
\hline$H^{m}(\Omega)$ & $\mathrm{W}^{\mathrm{m}, \mathrm{p}}(\Omega)$, for $\mathrm{p}=2$ \\
\hline$L^{p}(\Omega)$ & $W^{0, p}(\Omega)$ \\
\hline
\end{tabular}

\section{Mathematical Analysis for Steady Transport Problem}

Let $\Omega$ is a bounded, open and connected Lipschitz domain of $\mathbb{R}^{d}, d=2,3$. In this domain, we consider the steady tensorial transport equation which is of hyperbolic type, defined by

$$
\boldsymbol{\sigma}+\lambda \mathbf{u} \cdot \nabla \boldsymbol{\sigma}=\mathbf{g}
$$

where $\lambda \epsilon L^{\infty}(\Omega), u \epsilon L^{\infty}(\Omega)$ and $g \epsilon L^{\infty}(\Omega)$ are given.

In order to close this hyperbolic system, and to obtain a well-posed problem, the above equation need to be supplemented by the boundary conditions on inflow 
sections of the boundary, according to the hyperbolic partial differential equations (PDE) theory.

Componentwise, the equation (1) can be written as

$$
\begin{aligned}
& \sigma_{i j}+\lambda \mathbf{u} \cdot \nabla \sigma_{i j}=g_{i j}, \quad i, j=1,2 \\
& \Leftrightarrow \frac{1}{\lambda} \sigma_{i j}+\mathbf{u} \cdot \nabla \sigma_{i j}=\frac{1}{\lambda} g_{i j}, \lambda \neq 0
\end{aligned}
$$

Without loss of generality we can take $\lambda \neq 0$ because if $\lambda=0$, we have $\sigma=g$ and $g$ is given.

Assuming $\mu=1 / \lambda$, the componentwise equation is scalar and can be identified as the hyperbolic equation known as advection-reaction equation

$$
\mu \omega+\mathbf{u} \cdot \nabla \omega=h
$$

\subsection{Advection-reaction equation}

I consider the steady advection-reaction equation with inflow homogeneous boundary condition

$$
\begin{cases}\mu \omega+\mathbf{u} \cdot \nabla \omega=h \text { in } \Omega \\ \mathbf{u}=0 & \text { on } \partial \Omega^{-}\end{cases}
$$

where $\mu \in L^{\infty}(\Omega)$ is the reaction coefficient, $\mathbf{u} \in \mathbf{L}^{\infty}(\Omega)$ is the advective velocity field, $h \in L^{2}(\Omega)$ is the source term, $\omega$ is the unknown scalar function and $\partial \Omega^{-}$denotes the inflow part of the boundary of $\Omega$, namely

$\partial \Omega^{-}=\{\mathbf{x} \in \partial \Omega: \mathbf{u}(\mathbf{x}) \cdot \mathbf{n}(\mathbf{x})<0\}$

with $n=\left(n^{1},-----n^{d}\right)^{t}$ be a unit outward normal to $\partial \Omega$.

In the similar way, we define the outflow part of $\partial \Omega$ as

$\partial \Omega^{+}=\{\mathbf{x} \in \partial \Omega: \mathbf{u}(\mathbf{x}) \cdot \mathbf{n}(\mathbf{x})>0\}$

and the interior of the set $\{\mathbf{x} \in \partial \Omega: \mathbf{u}(\mathbf{x}) \cdot \mathbf{n}(\mathbf{x})=0\}$ as

$$
\partial \Omega^{0}=\partial \Omega \backslash\left(\overline{\partial \Omega^{-}} \cup \overline{\partial \Omega^{+}}\right) .
$$

I assume that the inflow and outflow boundaries are well separated, i.e.,

$$
\operatorname{dist}\left(\partial \Omega^{-}, \partial \Omega^{+}\right)>0 \text {. }
$$

I assume the following additional hypothesis on $\mu$ :

there exists $\mu_{0}$ such that

$$
\mu(\mathbf{x})-\frac{1}{2} \nabla \cdot \mathbf{u}(\mathbf{x}) \geq \mu_{0}>0 \quad \text { a.e.in } \Omega \text {. }
$$


To obtain the weak formulation of (4) I introduce the graph space

$$
W=\left\{\omega \in L^{2}(\Omega): \mathbf{u} \cdot \nabla \omega \in L^{2}(\Omega)\right\} \subset L^{2}(\Omega)
$$

\section{Lemma 1:}

$W$ is a Hilbert space with respect to the graph norm

$$
\|\omega\|_{W}=\|\omega\|_{L^{2}(\Omega)}+\|\mathbf{u} \cdot \nabla \omega\|_{L^{2}(\Omega)} .
$$

Here $W$ is dense in $L^{2}(\Omega)$ and $H^{1}(\Omega)$ is subspace of $W$.

Lesaint [4] guarantees the solution of (4) in the following results that he proves. The following theorem guarantees the existence and uniqueness of the solution of the approach problem.

\section{Theorem 1:}

Assume that $\mu \in L^{\infty}(\Omega)$ and $h \in L^{2}(\Omega)$. Then problem (4) has a unique strong solution $u \in W$.

To specify mathematically the meaning of the boundary condition, we need to define the trace on $\partial \Omega$ of function in $W$. For that, we introduce the real-valued functions which are square integrable with respect to the measure $|\mathbf{u . n}| d s$, where $d s$ is the Lebesgue measure on $\partial \Omega$, i.e.,

$$
L^{2}(\partial \Omega ;|\mathbf{u} . \mathbf{n}|)=\left\{v \text { is measurable on } \partial \Omega: \int_{\partial \Omega}|\mathbf{u} . \mathbf{n}| v^{2} d s<\infty\right\} .
$$

Introducing the following bilinear form which is continuous and $L^{2}$-coercive in $\mathrm{W} \mathbf{x}$ W:

$$
a(\omega, v)=\int_{\Omega} \mu v \omega+\int_{\Omega}(\mathbf{u} \cdot \nabla \omega) v+\int_{\partial \Omega} \frac{1}{2}(|\mathbf{u} \cdot \mathbf{n}|-\mathbf{u} \cdot \mathbf{n}) \omega v \quad \forall \omega, v \in W
$$

We obtain the following variational formulation of the advective-reaction problem:

$$
\left\{\begin{array}{l}
\text { Find } \omega \in W \text { such that } \\
a(\omega, v)=(h, v) \forall v \in W
\end{array}\right.
$$

Problem (10) is well-posed which can be proved as the consequence of the LaxMilgram theorem. The details of the Lax-Milgram theorem is reported elsewhere [5].

Problem (10) is the variational problem of (4). Moreover, if $\omega \in W$ is the solution of (10), then

$$
\begin{array}{r}
\mu \omega+\mathbf{u} \cdot \nabla \omega=h \text { a.e. in } \Omega \\
\mu=0 \text { a.e. in } \Omega
\end{array}
$$

i.e., $\omega$ is a weak solution of (4). 


\section{Discontinuous Galerkin Method}

The first discontinuous Galerkin method for hyperbolic partial differential equations have been introduced in 1973 by Reed and Hill to simulate nutron transport problem. The analysis of abstract form for this discrete problem was done one year later by Lesaint and Raviart [4]. More recently, the discontinuous Galerkin method for hyperbolic equations had a significant development based on numerical fluxes [7]. Discontinuous Galerkin method can be viewed as finite element method [8] with relaxed continuity at interelement boundaries. The essential idea of the method is derived from the fact that the shape functions can be chosen so that the field variable and/or its derivatives are discontinuous across the element boundaries [9]. The effect of the boundary conditions are gradually propagate through element-by-element connection. In this way it is possible to introduce a centering in a scheme that contains the integral over the edges, using the right and left values of the edge side, along the direction of flow.

\subsection{Discrete transport problem}

The details of this method can be found in literature [2-4]. We consider $\Omega$ a polyhedra, because in this way we can cover exactly a mesh of polyhedral elements.

Let $\left\{\tau_{h}\right\}_{h>0}$ be a family of non-degenerate, regular triangulation of $\Omega$. An edge $F$ of $\bar{\Omega}$ is a mesh face. Let $F_{h}^{i}$ be the set of interfaces and $F_{h}^{b}$ be the set of boundary faces, and we set

$F_{h}=F_{h}^{i} \cup F_{h}^{b}$

For all $F \in F_{h}^{i}$ and i.e. $x \in F$, the average and jump of a scalar function is defined, respectively, as

$$
\{v\}_{F}(x)=\frac{v_{\mid K_{1}}(x)+v_{\mid K_{2}}(x)}{2},[v]_{F}(x)=v_{\mid K_{1}}(x)-v_{\mid K_{2}}(x)
$$

where $K_{i}, i=1,2$ are the distinct mesh elements such that $F=\partial K_{1} \cap \partial K_{2}$

\subsection{Approach problem}

In the framework of the transport problem, I denote by $S_{1}$ the subspace of $L^{2}(\Omega)$ whose functions are piecewise linear polynomial functions over $\tau_{h}$ with degree less or equal to 1 .

If the exact solution $\omega$ is regular, we hope that the approach solution will be regular also. In this sense, we added to the classical formulation a priori small term

$$
\int_{\partial K}\left(\alpha\left|\mathbf{u} \cdot \mathbf{n}_{K}\right|-\frac{1}{2} \mathbf{u} \cdot \mathbf{n}_{K}\right)\left[\omega_{h}\right] v_{h}
$$

expressing the discontinuities of the solution approach to interfaces of elements.

So the approach formulation of the advective-reaction problem is defined as: 
find $\omega_{h} \in S_{1}: \omega_{h}=g_{h}$ on $\partial \Omega^{-}$such that

$$
\begin{aligned}
& \sum_{K \in \tau_{h}} \int_{K}\left(\mu \omega_{h}+\mathbf{u} \cdot \nabla \omega_{h}\right) v_{h}+\int_{\partial K}\left(\alpha\left|\mathbf{u} \cdot \mathbf{n}_{K}\right|-\frac{1}{2} \mathbf{u} \cdot \mathbf{n}_{K}\right)\left[\omega_{h}\right] v_{h} \\
& -\int_{\partial \Omega^{-}}\left|\mathbf{u} \cdot \mathbf{n}_{K}\right| \omega_{h} \chi_{\partial \Omega^{-}} v_{h}=\int_{K} h v_{h}, \forall v_{h} \in S_{1}: v_{h}=0 \text { on } \partial \Omega^{-}
\end{aligned}
$$

where $g_{h}$ is an approach of $g$ on $\partial \Omega^{-}, \mathbf{n}_{K}$ is the unit outward normal to $K$ and $\chi_{\partial \Omega^{-}}$ denotes the characteristic function of $\partial \Omega^{-}$.

The parameter $\alpha$ can vary from face to face but the value $\alpha=1 / 2$ is used usually according to the literature. With this value, one obtains the DG method analyzed by Lesaint and Raviart [9]. In this case the term $\left(\alpha\left|\mathbf{u} \cdot \mathbf{n}_{K}\right|-\frac{1}{2} \mathbf{u} \cdot \mathbf{n}_{K}\right)\left[\omega_{h}\right]$ is non zero only that part of the boundary $\partial K$ where $\mathbf{u} \cdot \mathbf{n}_{K}<0$.

\section{Numerical Results and Discussions}

In this section, we are interested in the implementation of the iterative method based on the application of a fixed point algorithm in FreeFem+ to solve the transport equation. We develop our own script in FreeFem++ to obtain the numerical solution of the non-dimensional steady tensorial transport equation using $\mathbb{P}_{1}$ discontinuous $\left(\mathbb{P}_{1} d c\right)$ finite element. For this type of elements, due to interpolation problem, FreeFem++ doesn't consider the degree of freedom as the vertices but three vertices move inside on the element with the linear map $T(X)=G+0.99(X-G)$ where $G$ is the barycenter. In this way, the number of degree of freedom is 3 times of the number of elements.

We consider the following non-dimensional steady tensorial transport problem defined in squared domain $\Omega=[0,1]^{2}$ :

find $\boldsymbol{\sigma} \in \mathbf{L}^{2}(\Omega)$ such that

$$
\boldsymbol{\sigma}+W e[(\mathbf{u} \cdot \nabla) \boldsymbol{\sigma}]=2 \lambda \mathbf{D}(\mathbf{u})+W e\left[(\nabla \mathbf{u}) \boldsymbol{\sigma}+\boldsymbol{\sigma}(\nabla \mathbf{u})^{t}\right] \text { in } \Omega
$$

where $W e$ is a dimensionless number known as Weissenberg number and $\mathbf{D}(\mathbf{u})=\frac{1}{2}\left[\nabla \mathbf{u}+(\nabla \mathbf{u})^{t}\right]$ is the symmetric part of the velocity gradient. Small values of We means that the fluid is little elastic.

We suppose that the vector field $u$ is given by [10]

$$
\mathbf{u}(x, y)=\left(\left(x^{2}-x\right)^{2}\left(y^{2}-y\right)(2 y-1),-\left(x^{2}-x\right)\left(y^{2}-y\right)^{2}(2 x-1)\right)
$$

Using the discrete formulation, the dimensionless transport problem can be written in terms of its components as a system of three scalar transport equations (advectionreaction equations) of three stress tensor components $\sigma_{11}, \sigma_{12}$, and $\sigma_{22}$. ( $\sigma$ is symmetric $\left.\left(\sigma_{12}=\sigma_{21}\right)\right)$, i.e., the dimensionless transport problem is, find $\left(\sigma_{11}, \sigma_{12}, \sigma_{22}\right)$ such that 


$$
\left\{\begin{array}{c}
\int_{\Omega}\left[\sigma_{h, 11}+W e\left(u_{1} \frac{\partial \sigma_{h, 11}}{\partial x_{1}}+u_{2} \frac{\partial \sigma_{h, 11}}{\partial x_{2}}\right)\right] \tau_{h, 11}+W e \int_{\partial K}\left(\alpha\left|\mathbf{u} \cdot \mathbf{n}_{K}\right|-\frac{1}{2} \mathbf{u} \cdot \mathbf{n}_{K}\right)\left[\sigma_{h, 11}\right] \tau_{h, 11} \\
=2 \lambda \int_{\Omega} \frac{\partial u_{1}}{\partial x_{1}} \tau_{h, 11}+2 W e \int_{\Omega}\left[\frac{\partial u_{1}}{\partial x_{1}} \sigma_{h, 11}+\frac{\partial u_{1}}{\partial x_{2}} \sigma_{h, 12}\right] \tau_{h, 11} \\
\int_{\Omega}\left[\sigma_{h, 12}+W e\left(u_{1} \frac{\partial \sigma_{h, 12}}{\partial x_{1}}+u_{2} \frac{\partial \sigma_{h, 12}}{\partial x_{2}}\right)\right] \tau_{h, 12}+W e \int_{\partial K}\left(\alpha\left|\mathbf{u} \cdot \mathbf{n}_{K}\right|-\frac{1}{2} \mathbf{u} \cdot \mathbf{n}_{K}\right)\left[\sigma_{h, 12}\right] \tau_{h, 12} \\
=\lambda \int_{\Omega}\left(\frac{\partial u_{1}}{\partial x_{2}}+\frac{\partial u_{2}}{\partial x_{1}}\right) \tau_{h, 12}+W e \int_{\Omega}\left[\frac{\partial u_{2}}{\partial x_{1}} \sigma_{h, 11}+\left(\frac{\partial u_{1}}{\partial x_{2}}+\frac{\partial u_{2}}{\partial x_{1}}\right) \sigma_{h, 12}+\frac{\partial u_{1}}{\partial x_{2}} \sigma_{h, 22}\right] \tau_{h, 12} \\
\int_{\Omega}\left[\sigma_{h, 22}+W e\left(u_{1} \frac{\partial \sigma_{h, 22}}{\partial x_{1}}+u_{2} \frac{\partial \sigma_{h, 22}}{\partial x_{2}}\right)\right] \tau_{h, 22}+W e \int_{\partial K}\left(\alpha\left|\mathbf{u} \cdot \mathbf{n}_{K}\right|-\frac{1}{2} \mathbf{u} \cdot \mathbf{n}_{K}\right)\left[\sigma_{h, 22}\right] \tau_{h, 22} \\
=2 \lambda \int_{\Omega} \frac{\partial u_{2}}{\partial x_{2}} \tau_{h, 22}+2 W e \int_{\Omega}\left[\frac{\partial u_{2}}{\partial x_{1}} \sigma_{h, 12}+\frac{\partial u_{2}}{\partial x_{2}} \sigma_{h, 22}\right] \tau_{h, 22}
\end{array}\right.
$$

I use a fixed point iterative method where we obtain the component of $\sigma^{n+1}$ by the value of the previous iteration $\sigma^{n}$. This iterative method can be described as follows: given $\left(\sigma_{11}^{0}, \sigma_{12}^{0}, \sigma_{22}^{0}\right)$ such that

$$
\left\{\begin{array}{c}
\int_{\Omega}\left[\sigma_{h, 11}^{n+1}+W e\left(u_{1} \frac{\partial \sigma_{h, 11}^{n+1}}{\partial x_{1}}+u_{2} \frac{\partial \sigma_{h, 1}^{n+1}}{\partial x_{2}}\right)\right] \tau_{h, 11}+W e \int_{\partial K}\left(\alpha\left|\mathbf{u} \cdot \mathbf{n}_{K}\right|-\frac{1}{2} \mathbf{u} \cdot \mathbf{n}_{K}\right)\left[\sigma_{h, 11}^{n+1}\right] \tau_{h, 11} \\
=2 \lambda \int_{\Omega} \frac{\partial u_{1}}{\partial x_{1}} \tau_{h, 11}+2 W e \int_{\Omega}\left[\frac{\partial u_{1}}{\partial x_{1}} \sigma_{h, 11}^{n}+\frac{\partial u_{1}}{\partial x_{2}} \sigma_{h, 12}^{n}\right] \tau_{h, 11} \\
\int_{\Omega}\left[\sigma_{h, 12}^{n+1}+W e\left(u_{1} \frac{\partial \sigma_{h, 12}^{n+1}}{\partial x_{1}}+u_{2} \frac{\partial \sigma_{h, 12}^{n+1}}{\partial x_{2}}\right)\right] \tau_{h, 12}+W e \int_{\partial K}\left(\alpha\left|\mathbf{u} \cdot \mathbf{n}_{K}\right|-\frac{1}{2} \mathbf{u} \cdot \mathbf{n}_{K}\right)\left[\sigma_{h, 12}^{n+1}\right] \tau_{h, 12} \\
=\lambda \int_{\Omega}\left(\frac{\partial u_{1}}{\partial x_{2}}+\frac{\partial u_{2}}{\partial x_{1}}\right) \tau_{h, 12}+W e \int_{\Omega}\left[\frac{\partial u_{2}}{\partial x_{1}} \sigma_{h, 11}^{n}+\left(\frac{\partial u_{1}}{\partial x_{1}}+\frac{\partial u_{2}}{\partial x_{2}}\right) \sigma_{h, 12}^{n}+\frac{\partial u_{1}}{\partial x_{2}} \sigma_{h, 22}^{n}\right] \tau_{h, 12} \\
\int_{\Omega}\left[\sigma_{h, 22}^{n+1}+W e\left(u_{1} \frac{\partial \sigma_{h, 22}^{n+1}}{\partial x_{1}}+u_{2} \frac{\partial \sigma_{h, 22}^{n+1}}{\partial x_{2}}\right)\right] \tau_{h, 22}+W e \int_{\partial K}\left(\alpha\left|\mathbf{u} \cdot \mathbf{n}_{K}\right|-\frac{1}{2} \mathbf{u} \cdot \mathbf{n}_{K}\right)\left[\sigma_{h, 22}^{n+1}\right] \tau_{h, 22} \\
=2 \lambda \int_{\Omega} \frac{\partial u_{2}}{\partial x_{2}} \tau_{h, 22}+2 W e \int_{\Omega}\left[\frac{\partial u_{2}}{\partial x_{1}} \sigma_{h, 12}^{n}+\frac{\partial u_{2}}{\partial x_{2}} \sigma_{h, 22}^{n}\right] \tau_{h, 22}
\end{array}\right.
$$

For the computational implementation, we consider a mesh $100 \times 100$ with 20000 elements and $60000 \mathbb{P}_{1} d c$ nodes which is shown in Fig. 1 take $\lambda=0.1,0.5$ and 0.9 .

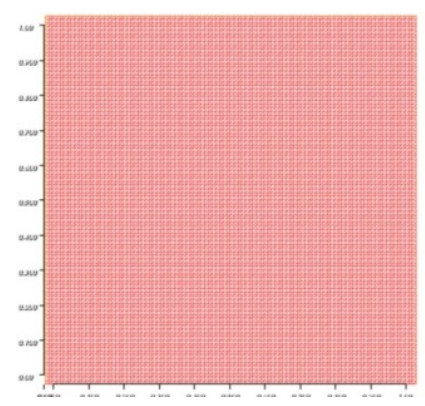

Fig. 1. Meshes over the square $[0,1]^{2}$. 
For each $\lambda$, I do the study for different values of Weissenberg number $W e$ admissible for convergence of iterative method.

The linear system was solved using the default solver sparse-solver [1] and the details are available in literature [11].

The numerical solutions are illustrated both graphically and computationally (with color scales) in Figs. 2-10 for different Weissenberg number $W e$ and $\lambda$. All the color scales are defined for 20 values equally spaced between its minimum (below) and the maximum (top).

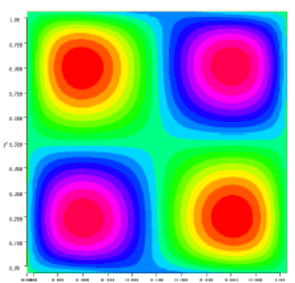

(a) $\sigma_{11}$

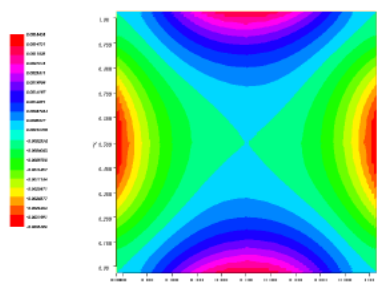

(b) $\sigma_{12}$

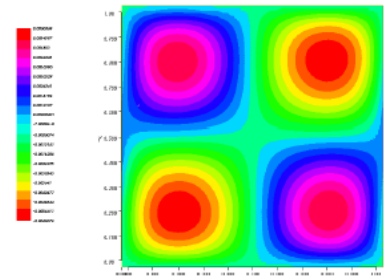

(c) $\sigma_{22}$

Fig. 2. Contours of the stress tensor components at $\mathrm{We}=1$ and $\lambda=0.1$.

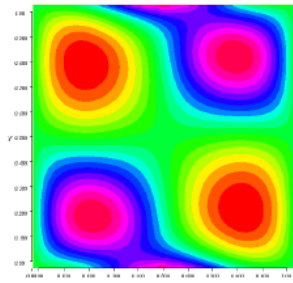

(a) $\sigma_{11}$
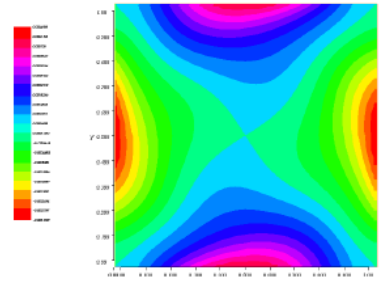

(b) $\sigma_{12}$
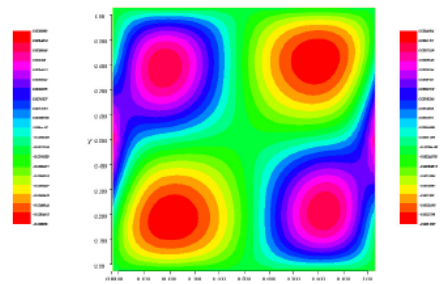

(c) $\sigma_{22}$

Fig. 3. Contours of the stress tensor components at $\mathrm{We}=5$ and $\lambda=0.1$.

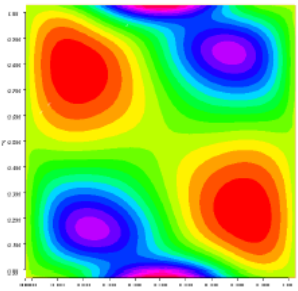

(a) $\sigma_{11}$
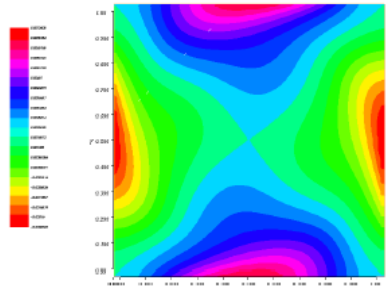

(b) $\sigma_{12}$
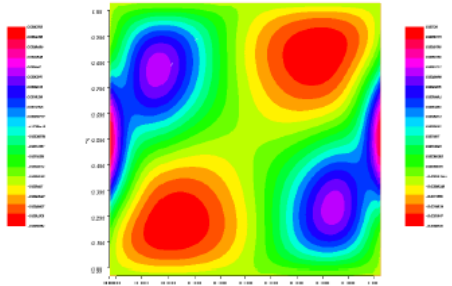

(c) $\sigma_{22}$

Fig. 4. Contours of the stress tensor components at $\mathrm{We}=10$ and $\lambda=0.1$. 


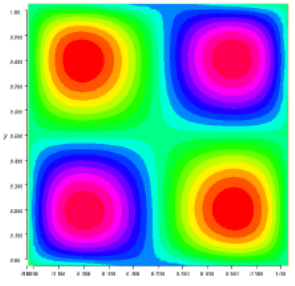

(a) $\sigma_{11}$
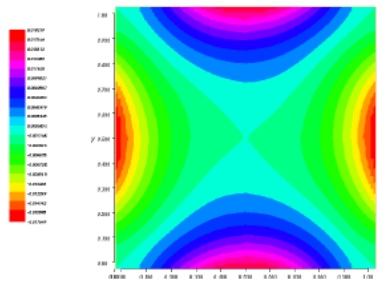

(b) $\sigma_{12}$
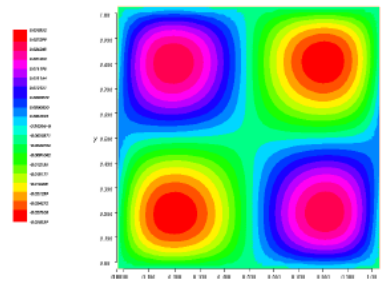

(c) $\sigma_{22}$

Fig. 5. Contours of the stress tensor components at $\mathrm{We}=1$ and $\lambda=0.5$.

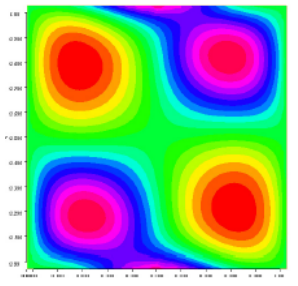

(a) $\sigma_{11}$
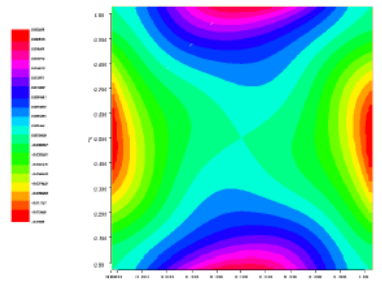

(b) $\sigma_{12}$
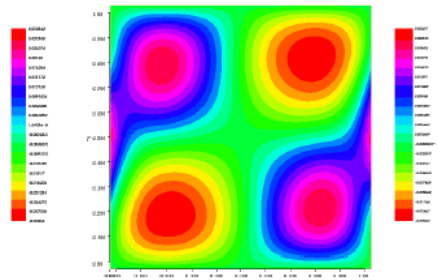

(c) $\sigma_{22}$

Fig. 6. Contours of the stress tensor components at $\mathrm{We}=5$ and $\lambda=0.5$.

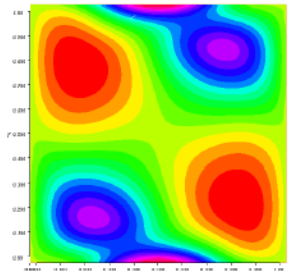

(a) $\sigma_{11}$
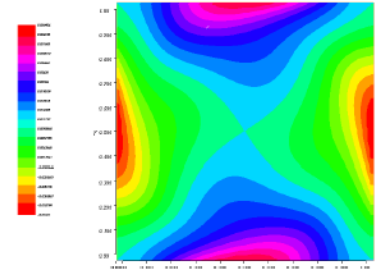

(b) $\sigma_{12}$
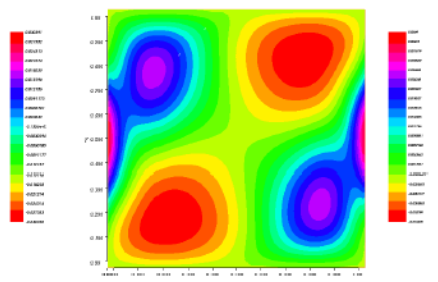

(c) $\sigma_{22}$

Fig. 7. Contours of the stress tensor components at $\mathrm{We}=10$ and $\lambda=0.5$.

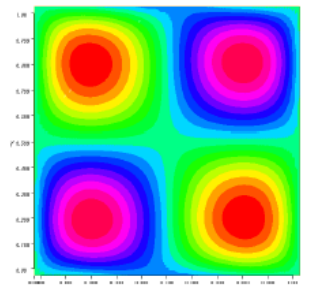

(a) $\sigma_{11}$

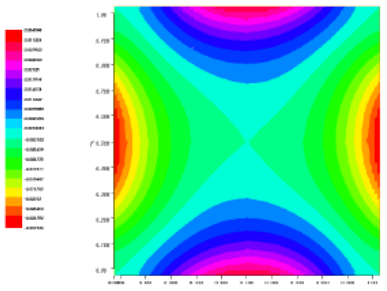

(b) $\sigma_{12}$

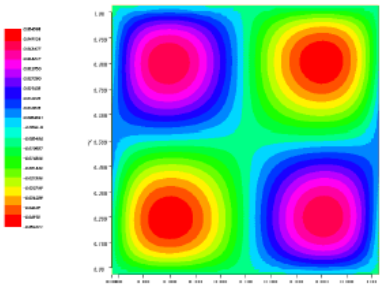

(c) $\sigma_{22}$

Fig. 8. Contours of the stress tensor components at $\mathrm{We}=1$ and $\lambda=0.9$. 


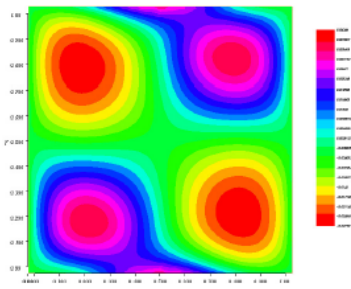

(a) $\sigma_{11}$

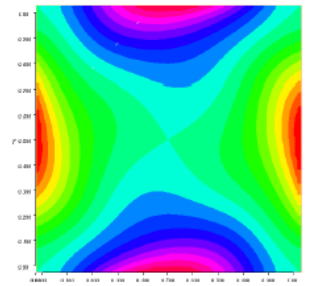

(b) $\sigma_{12}$
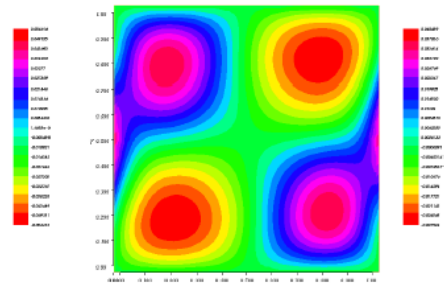

(c) $\sigma_{22}$

Fig. 9. Contours of the stress tensor components at $\mathrm{We}=5$ and $\lambda=0.9$.

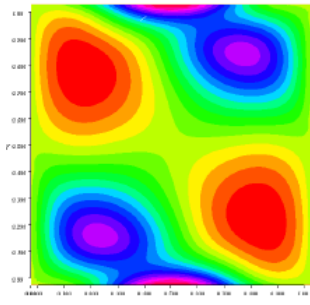

(a) $\sigma_{11}$
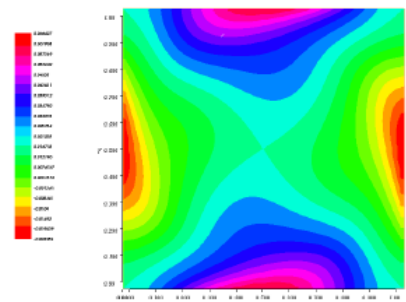

(b) $\sigma_{12}$
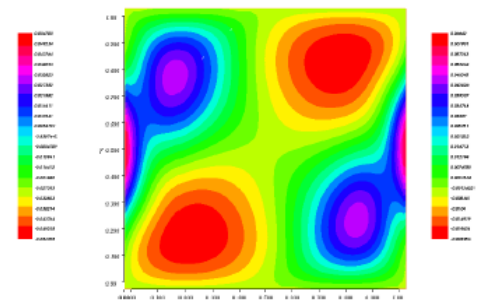

(c) $\sigma_{22}$

Fig. 10. Contours of the stress tensor components at $\mathrm{We}=10$ and $\lambda=0.9$.

I analyze the effects of Weissenberg number We. I compared the different solutions obtained for $W e$ between 1 and 10 with fixed $\lambda$. For each $\lambda$, I observed the occurrence of numerical instabilities associated with the increased $W e$ as we can see in the above Figures. When $\lambda$ increase, I observed that the qualitative behavior is same but quantitatively the amplitude of each component increases.

\section{Conclusion}

In this paper I have obtained the approximate solutions of steady tensorial transport equations using discontinuous Galerkin finite element method with an iterative fixed point algorithm implemented in FreeFem++. In fact, for known velocity, I have obtained the viscoelastic extra stress tensor which is caused for the elastic behavior of fluid particles in the fluid motion satisfying the transport equations. The numerical results are obtained by considering the benchmark problem over a domain of unit square. I have represented the solutions both graphically and computationally, and also have observed the behavior of the solutions of the results. I have obtained the behavior of the three stress tensor components for different Weissenberg number $W e$ and $\lambda$. I observed that when we increase $W e$ for same $\lambda$, some perturbations are appeared. These perturbations are the numerical perturbations related with the high value of $W e$. Taking different $\lambda$, I have observed the same behavior when the $W e$ was increased. Comparing these behaviors with the previous behaviors, I obtained, more or 
less, the same qualitative behaviors. But the quantitative behaviors increase. The maximum value increases and the minimum value decreases, and the rate of perturbation is becoming greater. This is, in fact, the elastic behavior of the fluid for high $W e$ which coincides with the known phenomena that the high We lead a numerical instabilities which can be seen in the behavior of numerical solutions or lead the divergence of algorithm. For further work, we can try to solve the transport problem for bigger Weissenberg number to find the viscoelastic extra stress tensor.

Finally, it has been established that FreeFem++ is capable of providing the better numerical approximations of the viscoelastic extra stress tensor by solving hyperbolic transport equations.

\section{References}

1. F. Hecht, New Development in Freefem++, J. Numer. Math. 20(3-4), 251 (2012).

2. A. Ern and J. Guermond, SIAM J. Numer. Anal. 44(2), 753 (2006). http://dx.doi.org/10.1137/050624133

3. B. Q. Li , Discontinuous Finite Elements in Fluid Dynamics and Heat Transfer (Springer Verlag, 2006).

4. P. Lesaint and P. A. Raviart, On a Finite Element Method for Solving the Neutron Transport Equation, ed. C. Boor, Mathematical Aspects of Finite Elements in Partial Differential Equations, (New York, Academic press, 1974) pp. 89-123.

5. H. Brezis, Functional Analysis, Sobolev Spaces and Partial Differential Equations (Springer, 2011).

6. R. A. Adams and J. F. Fournier, Sobolev Space, $2^{\text {nd }}$ Edition (Academic Press, New York, 2003).

7. B. Cockburn, G. Karniadakis, and C. Shu, Discontinuous Galerkin Methods-theory, Computation and Application, Lecture Notes in Computer Science and Engineering (Springer, 2000) 11.

8. E. Becker, G. Carey, and J. Oden, Finite Elements. An Introduction (Prentice Hall, Inc., Englewood Cliffs, New Jersey, 1981) 1.

9. T. Bodnar, M. Pires, and J. Janela, Math. Model. Nat. Phenom. 9(6), 117 (2014). http://dx.doi.org/10.1051/mmnp/20149609

10. L. Borges, PhD Thesis, IST, Lisbon (2006).

11. Y. Saad, Iterative Methods for Sparse Linear Systems, $2^{\text {nd }}$ Edition (Society for Industrial and Applied Mathematics, Philadelphia, 2003). http://dx.doi.org/10.1137/1.9780898718003 